\title{
Exposure to nanoparticles is related to pleural effusion, pulmonary fibrosis and granuloma
}

\author{
Y. Song*, X. Lï and X. Du*
}

ABSTRACT: Nano materials generate great benefits as well as new potential risks. Animal studies and in vitro experiments show that nanoparticles can result in lung damage and other toxicity, but no reports on the clinical toxicity in humans due to nanoparticles have yet been made.

The present study aimed to examine the relationship between a group of workers' presenting with mysterious symptomatic findings and their nanoparticle exposure.

Seven young female workers (aged 18-47 yrs), exposed to nanoparticles for 5-13 months, all with shortness of breath and pleural effusions were admitted to hospital. Immunological tests, examinations of bacteriology, virology and tumour markers, bronchoscopy, internal thoracoscopy and video-assisted thoracic surgery were performed. Surveys of the workplace, clinical observations and examinations of the patients were conducted.

Polyacrylate, consisting of nanoparticles, was confirmed in the workplace. Pathological examinations of patients' lung tissue displayed nonspecific pulmonary inflammation, pulmonary fibrosis and foreign-body granulomas of pleura. Using transmission electron microscopy, nanoparticles were observed to lodge in the cytoplasm and caryoplasm of pulmonary epithelial and mesothelial cells, but are also located in the chest fluid. These cases arouse concern that long-term exposure to some nanoparticles without protective measures may be related to serious damage to human lungs.

KEYWORDS: Foreign-body granuloma, human, hypoxaemia, pleural effusion, polyacrylate nanoparticle, pulmonary fibrosis

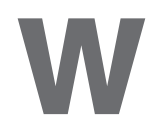
ith the explosion of nano materials, the benefits afforded by them are expected to have significant impacts on almost all industries and all areas of society. New materials based on nanotechnology are already reaching the market in a wide variety of consumer products. It is estimated that the nanotechnology economy will exceed $\$ 1$ trillion by 2012 [1]. However, the vastness and novelty of nano materials leaves open the possibility that they may generate new risks to workers, consumers, and the environment. Several previous studies caution against the possible significant adverse effects of nano materials [2-5].

Animal studies and in vitro experiments show that nanoparticles could result in lung damage and other toxicity. PAPAGEORgIOU et al. [6] showed that cobalt-chromium alloy nanoparticles caused more free radicals and induced more DNA damage, more aneuploidy and more cytotoxicity than micron-sized particles in human fibroblasts in tissue culture. In a mice study [7], it was demonstrated that pharyngeal aspiration of single-walled carbon nanotubes elicited pulmonary effects: acute inflammation with early onset and progressive fibrosis, granulomas and functional respiratory deficiencies. A study on carbon nanoparticle [8] exposure in both in vitro and in vivo systems, presents evidence that nanoparticles, accumulated in the plasma membranes of cells, increased alveolar macrophage function with antigen presentation, significantly exacerbated airway hyperresponsiveness, and caused an influx of macrophages into the lungs.

It is possible, theoretically, that long-term exposure to nanoparticles may cause serious damage to humans as well as animals. However, no reports on the clinical toxicity in humans due to long-term exposure to nanoparticles have been made until now. We report on a group of patients

\section{AFFILIATIONS}

Depts of * Occupational Medicine and Clinical Toxicology, and \#Pathology, Beijing Chaoyang Hospital, Capital University of Medical Sciences, Beijing, China.

CORRESPONDENCE

Y. Song

Dept of Occupational Medicine and

Clinical Toxicology

Beijing Chaoyang Hospital

No. 8

Baijiazhuang Road

Chaoyang District

Beijing

China (100020)

E-mail: songrain123@hotmail.com

Received:

Nov 282008

Accepted after revision:

May 112009 
with mysterious findings, pleural effusions, pulmonary fibrosis, foreign-body granulomas and hypoxaemia, which may be the result of toxicity from long-term exposure to nanoparticles.

\section{METHODS \\ Patients}

From January 2007 to April 2008, seven young female patients, working in the same department in a print plant, were admitted to our hospital (Beijing Chaoyang Hospital, Beijing, China). They suffered from the same symptoms: shortness of breath, and the same clinical findings of pleural effusion and pericardial effusion. These females previously underwent treatment in local hospitals including multiple thoracentesis attempts with recurrence of effusion, antibiotics, anti-tuberculosis drugs, and methylprednisolone or prednisolone. All the patients were previously in good health, and denied any history of smoking or prior occupational exposure to hazardous materials; they used to do some housework or farmwork as peasants.

\section{Clinical examinations}

All examinations were approved by our hospital's ethics commission and informed consent was obtained before all the examinations and procedures. Routine tests of blood, urine, pleural fluid and arterial blood gas analysis were performed. Bacteriologic examinations, including cultures of blood, urine, sputum and fluid in chest cavity, were performed. The antibodies of Mycoplasma pneumoniae (immunoglobulin (Ig) G and IgM), Chlamydia pneumoniae (IgM), Legionella (IgG and IgM) and tubercle bacillus were detected by ELISA methods. Virology examinations were conducted including antibodies of Cytomegalovirus, Epstein-Barr virus, human syncytial virus, cache valley virus, rhinovirus, adenovirus, hepatitis virus (A, $\mathrm{B}, \mathrm{C}$ and E) and HIV. Positive antibody results were subsequently tested using PCR magnification. Immunological tests, including cell immunity (CD3, CD4 and CD8), humoral immunity (IgA, G, D, M, E, C3 and C4) and autoimmune autoantibodies (anti-nuclear antibody, anti-ribonucleic acid antibody, anti-nucleosome antibody, anti-skin-sensitising antibodies A and B, anti-SCL-70 antibody, anti histone antibody, rheumatoid factor, C-reacting protein and antistreptolysin-O test), were conducted. Imaging included radiographs of the chest, ultrasound of the thoracic cavity, heart, liver, kidney and spleen, and either computed tomography (CT) scanning of the chest or a CT pulmonary angiogram. Functional examinations included liver function assays, renal function tests and pulmonary function tests. Patients underwent a variety of procedures according to their symptoms including drainage of pleural effusions, bronchoscopic examinations with bronchoalveolar lavage (BAL) and transbronchial lung biopsy (TBLB), internal thoracoscopic examinations with lung biopsy, and video-assisted thoracic surgery (VATS) with wedge resection. Pathological examinations included light microscopy and transmission electron microscopy (TEM) of the pleura, pleural fluid and lung tissue.

\section{Survey of the workplace}

Epidemiologists from the Chinese Center of Disease Control (CDC), Beijing CDC, local CDC and doctors from our hospital carefully investigated the factory where the patients worked. The paste materials used by the patients and dust particles that accumulated in the local ventilation were analysed by gas chromatography/mass spectrometry (GC/MS).

\section{Treatment}

All patients underwent thoracentesis for both aetiological investigation of the pleural effusions and symptomatic relief. In three patients closed drainage of pleural effusions followed by thoracoscopy was performed, two of the seven patients underwent VATS. Antibiotics and supportive therapy, such as oxygen, were used due to persistent inflammation in the lungs, as demonstrated by chest radiographs and chest CT scans.

\section{RESULTS}

\section{Survey of the workplace}

A survey of the patients' workplace was conducted. It measures $\sim 70 \mathrm{~m}^{2}$, has one door, no windows and one machine which is used to air spray materials, heat and dry boards. This machine has three atomising spray nozzles and one gas exhauster (a ventilation unit), which broke 5 months before the occurrence of the disease. The paste material used is an ivory white soft coating mixture of polyacrylic ester.

Eight workers (seven female and one male) were divided into two equal groups each working $8-12 \mathrm{~h}$ shifts. Using a spoon, the workers took the above coating material (room temperature) to the open-bottom pan of the machine, which automatically air-sprayed the coating material at the pressure of 100-120 Kpa onto polystyrene (PS) boards (organic glass), which can then be used in the printing and decorating industry. The PS board was heated and dried at $75-100^{\circ} \mathrm{C}$, and the smoke produced in the process was cleared by the gas exhauster. In total, $6 \mathrm{~kg}$ of coating material was typically used each day. The PS board sizes varied from $0.5-1 \mathrm{~m}^{2}$ and $\sim 5,000 \mathrm{~m}^{2}$ were handled each workday. The workers had several tasks in the process including loading the soft coating material in the machine, as well as clipping, heating and handling the PS board. Each worker participated in all parts of this process.

Accumulated dust particles were found at the intake of the gas exhauster. During the 5 months preceding illness the door of the workspace was kept closed due to cold outdoor temperatures. The workers were all peasants near the factory, and had no knowledge of industrial hygiene and possible toxicity from the materials they worked with. The only personal protective equipment used on an occasional basis was cotton gauze masks. According to the patients, there were often some flocculi produced during air spraying, which caused itching on their faces and arms. It is estimated that the airflow or turnover rates of indoor air would be very slow, or quiescent due to the lack of windows and the closed door.

Of the eight workers, the seven females worked there for 513 months and all had the described symptoms. The lone male worker was employed for 3 months and was asymptomatic. The patients' family and the workers in other departments of the same plant did not have similar symptom complaints.

\section{Analysis of used paste and dust particles}

The used paste was analysed using GC/MS. The compound was stated to be polyacrylic ester by the paste producer. It contained the following components: butanoic acid, butyl 
ester, $N$-butyl ether, acetic acid, toluene, di-tert-butyl peroxide, 1-butanol, acetic acid ethenyl ester, isopropyl alcohol and ethylene dioxide. Electron microscopy of both the paste and the dust particles found nanoparticles $\sim 30 \mathrm{~nm}$ in diameter.

\section{General characteristics of the patients}

All the patients were young females, aged 18-47 yrs, average 29 yrs (table 1). Two were single and five were married. They had complaints of dyspnoea with exertion and signs consistent with pleural fluid and hypoxaemia. Four out of the seven patients were found to have hypoxaemia (oxygen tension $<10.7 \mathrm{kPa}$; normal range (NR) $10.7-13.3 \mathrm{kPa}$ ), but no carbon dioxide retention. Additionally, all patients suffered from a rash with intense itching on their faces, hands and forearms. All patients were found to have pleural fluid by ultrasound, and five patients had pericardial effusions at the depth of $\sim 4-8 \mathrm{~mm}$. There were no morphological abnormalities about the heart, liver, kidney and gynecologic organs.

\section{Results of routine blood and urine tests and functional tests of liver, kidney and lung}

All the patients had routine blood and urine tests. The results of the urine tests were normal. All the patients were noted to have white blood cell counts and red blood cell counts that were in the NR. Five patients had monocytosis with an elevated absolute monocyte count and neutropenia with a decreased absolute neutrophil count. Additionally, five patients had elevated erythrocyte sedimentation rate (ESR) and one patient had thrombocytopenia $\left(35-51 \times 10^{9} \cdot \mathrm{L}^{-1}, \mathrm{NR} 100-300 \times 10^{9} \cdot \mathrm{L}^{-1}\right)$.

After 7 months observation, six out of seven patients were found to have hypoproteinemia (albumin 25.5-28.9 $\mathrm{g} \cdot \mathrm{L}^{-1}$, NR 32.0-55.0 $\left.\mathrm{g} \cdot \mathrm{L}^{-1}\right)$, protein electrophoresis of serum showed decreased albumin (50.2-55.6\%, NR 60.0-71.0\%), and globulins were approximately in the NR $\left(\alpha_{1} 2.9 \%\right.$, NR $1.4-2.9 \% ; \alpha_{2} 15.4 \%$, NR 7.0-11.0\%; $\beta 14.1 \%$, NR 8.0-13\%; and $\gamma 12 \%$, NR 9.0-16.0\%). Functional tests of kidney were regularly conducted and within normal limits, but several patients were found with elevated levels of alanine aminotransferase (ALT) and aspartate aminotransferase (AST; table 2). Spirometry showed that all the patients suffered from small airway injury and restrictive ventilation dysfunction, and showed that three out of seven patients had severe lung damage: maximal vital capacity (VCmax; actual value/predicted value) $24.8-35.4 \%$
(NR $\geqslant 80 \%$ ); forced vital capacity (FVC) $24.6-35.1 \%$ (NR $\geqslant 80 \%$ ); and forced expiratory volume in $1 \mathrm{~s}$ (FEV1) $24.8-$ $36.5 \%$ predicted ( $\mathrm{NR} \geqslant 80 \%$ pred). One out of seven patients had moderate lung damage: VCmax $63.6 \%$; FVC $61.3 \%$; and FEV1 $63.4 \%$ pred; Three out of seven patients had mild lung damage: VCmax 71.6-92.6\%; FVC 75.6-91.9\%; and FEV1 60$70.6 \%$ pred.

\section{Immunological tests and aetiological examinations}

As for immunological tests for cell immunity, humoral immunity and autoimmunity, no significant clinical abnormities were found. As for bacterial investigation, the results of tests on acid-fast bacilli, M. pneumoniae and C. pneumoniae were all negative, except in two out of seven patients with positive results of $M$. pneumoniae (IgG (+) and $\operatorname{IgM}(+))$ and $C$. pneumoniae $(\operatorname{IgM}(+))$. However, there were no clinical correlations with the test results. As for virology examinations and the subsequently tests using PCR magnification on positive antibody results of virus, no clinical correlations were observed between the illness and the test results. Tumour markers, including $\alpha$ fetoprotein, carcinoembryonic-like antigen, neurone specific enolase and transferrin, were examined and all the results were negative both in serum and pleural fluid.

\section{Imaging studies}

Repeated examinations of chest radiographs and CT scanning showed all the patients to have pleural effusions; five patients had pericardial fluid, four out of seven patients had diffuse ground-glass opacities, six had pulmonary interstitial nodules, and three out of seven patients had lymph node adenopathies. No patients had pleural thickening, pleural nodules or pleural plaques. All the seven patients had pulmonary interstitial inflammation and pulmonary fibrosis. After 7 months observation, rapid progressive pulmonary interstitial fibrosis was observed in two patients (case 5 and case 1), also pleural calcification was observed in one patient (case 1), but in the other patients, pulmonary interstitial fibrosis developed very slowly.

\section{Thoracentesis and tests of pleural fluid}

The results of the pleural fluid analysis were similar for all the patients showing an amber exudate, which may be produced by physical or chemical irritation. The total cells were 30$485 \times 10^{6} \cdot \mathrm{L}^{-1}$; white blood cells were $10-470 \times 10^{6} \cdot \mathrm{L}^{-1}$, subsets of

\section{TABLE 1 Patients' characteristics}

\begin{tabular}{|c|c|c|c|c|c|c|c|c|c|c|}
\hline Case & Age yrs & Sex & $\begin{array}{l}\text { Duration of exposure } \\
\text { months }\end{array}$ & $\mathrm{PO}_{2} \mathrm{kPa}$ & $\begin{array}{l}\text { Pleural } \\
\text { effusion }\end{array}$ & $\begin{array}{l}\text { Pericardial } \\
\text { effusion }\end{array}$ & Bronchoscopy & Thoracoscopy & VATS & Outcome \\
\hline 1 & 29 & Female & 13 & 9.6 & Great & Small & + & + & - & Died \\
\hline 2 & 47 & Female & 11 & 10.5 & Great & None & + & + & - & Stable \\
\hline 3 & 18 & Female & 13 & 9.6 & Great & Small & + & + & + & Stable \\
\hline 4 & 29 & Female & 12 & 11.6 & Great & Small & + & - & - & Stable \\
\hline 5 & 19 & Female & 10 & 10.7 & Great & Small & + & - & + & Died \\
\hline 6 & 35 & Female & 10 & 12.9 & Great & Small & + & - & - & Stable \\
\hline 7 & 28 & Female & 5 & 12.9 & Small & None & + & - & - & Stable \\
\hline
\end{tabular}

$\mathrm{PO}_{2}$ : oxygen tension; VATS: video-assisted thoracic surgery. + : performed; -: not performed 


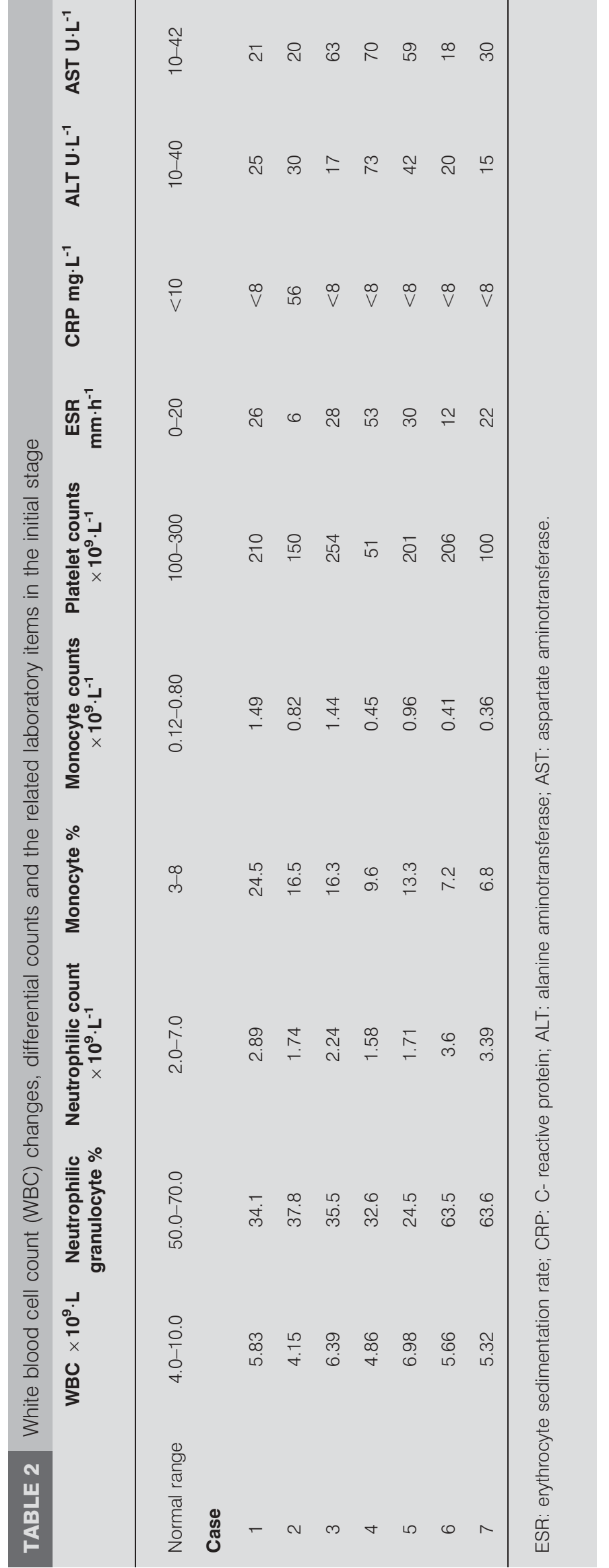

the cells were monocytes (55-85\%) and polykaryocytes (15$45 \%$ ), total protein was $40.1-49.3 \mathrm{~g} \cdot \mathrm{L}^{-1}$, and specific gravity was $>1.018$. Rivalta reaction, which is used as a puncture fluid test for differentiation of exudate and transudate, was negative. The concentrations of chloridion in all patients' pleural effusions $\left(108.0-111.8 \mathrm{mmol} \cdot \mathrm{L}^{-1}\right.$, NR $\left.118.0-129.0 \mathrm{mmol} \cdot \mathrm{L}^{-1}\right)$ were under the lower limit of normal, and concentrations of glucose in all patients' pleural effusions were elevated $\left(5.01-6.59 \mathrm{mmol} \cdot \mathrm{L}^{-1}\right.$, NR $2.50-4.44 \mathrm{mmol} \cdot \mathrm{L}^{-1}$ ). No malignant cells were found in the pleural fluid, mesothelial cells and lymphocytes were common.

\section{Bronchoscope inspection}

All patients had bronchoscopy, and four of them had swelling and congestion of the tunica mucosa bronchiorum.

\section{BAL fluid}

Examination of the BAL fluid (BALF) showed decreased macrophages $(29-79 \%$, NR $>84 \%)$, increased lymphocytes (15-54\%, NR $<13 \%)$, elevated neutrophil leukocytes $(6-18 \%$, $\mathrm{NR}<3 \%$ ), and eosinophils in the normal range $(0.5 \%$, NR $<0.5 \%$ ) in five of the patients. The other two patients were within the normal ranges for BALF cell counts. T-cells in the BALF were normal: CD3 69-73\% (NR 63-83\%), CD4 40\% (NR $40-70 \%$ ) and CD8 35\% (NR 20-40\%).

\section{TBLB}

The results of all patients were consistent showing aggregations of phagocytes and inflammatory cells, proteinaceous effusions in the alveolar space, swollen and widened alveolar septums with scattered neutrophil leukocytes, and pulmonary fibrosis (fig. 1a). The pathological examination of tunica mucosa bronchiorum showed the effusion of inflammatory cells (fig. 1b).

\section{Thoracoscopy}

Three out of the seven patients had thoracoscopy. Of these three patients, two patients had mild congestion of the partial pleura, but overall it was smooth and glossy with no neoplasm or exelcosis and one patient had a normal thoracoscopy. Biopsies of the pleura and fluid in the chest were also analysed. Pathological study of pleura showed foreign-body granulomas (fig. 1c) and haemorrhage, as well as fibrinous and inflammatory cells.

\section{VATS}

Two patients underwent VATS, one was an 18-yr-old female (case 3), 4 months after symptom onset. Wedge resection, lung biopsy and drainage of pleural effusions were performed. In total, $\sim 2,000 \mathrm{~mL}$ of fluid was drained from the chest cavity. Exploration of the chest cavity showed no pleural adhesions, capillary haemorrhage or air leak. On the lung surface, there were scattered small tubercles, which were biopsied from the superior, middle and inferior lobes. Pleural biopsy was also obtained.

There were nonspecific findings by light microscope such as fibrous thickening in the pleural membrane, pulmonary alveoli with emphysema-like alterations, widened alveolar septums, depositions of collagen fibres, engorged capillaries filled with lymphocytes and infiltrations of phagocytes in alveolar spaces.

The other patient who underwent VATS was a 19-yr-old female (case 5) 18 months after symptom onset. This was performed in an emergency due to respiratory failure, 

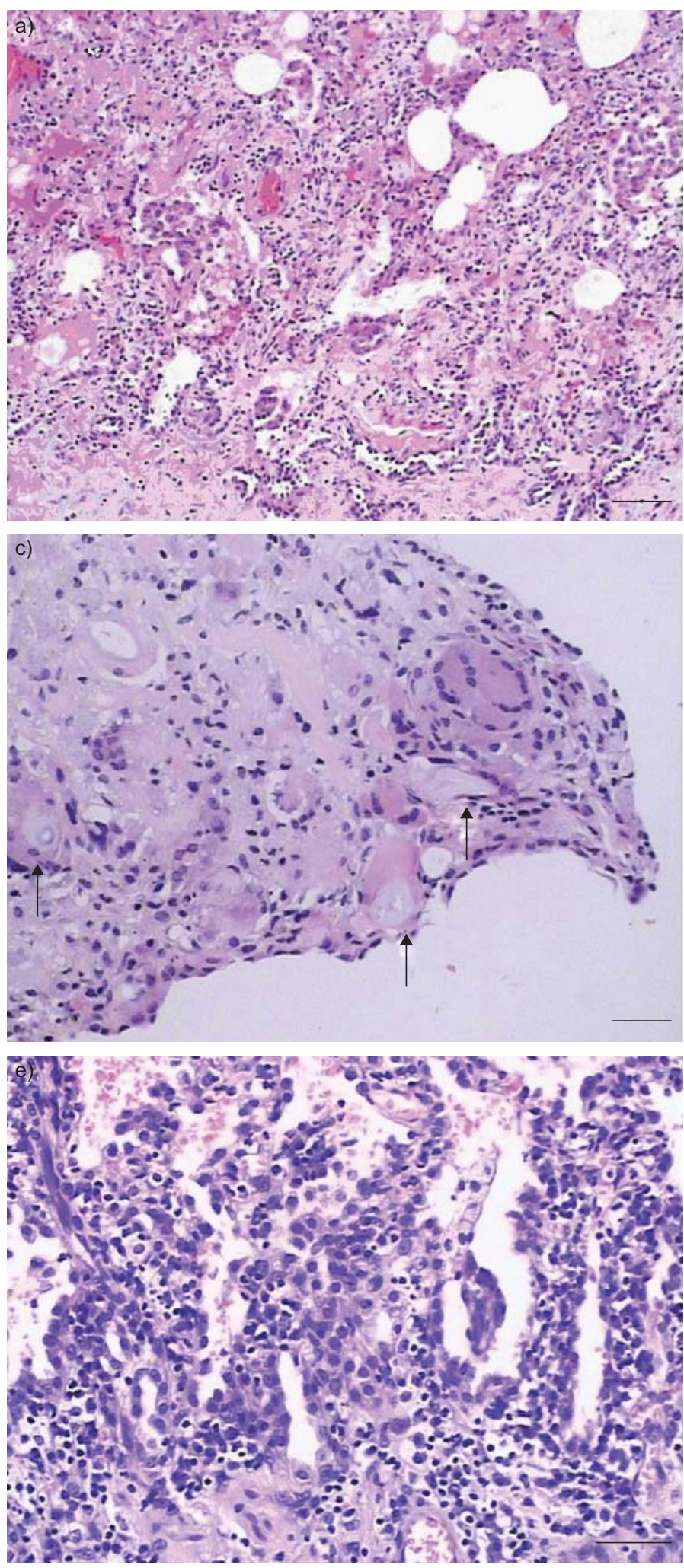

secondary to encapsulated pleural effusion formation, pulmonary fibrosis and severe pericardial effusion development. Moreover, she was in a very serious condition due to continuous closed drainage of the thoracic cavity and subsequent loss of proteins. Intraoperatively, multi-encapsulated pleural effusions and pleural fibrous lamina formations
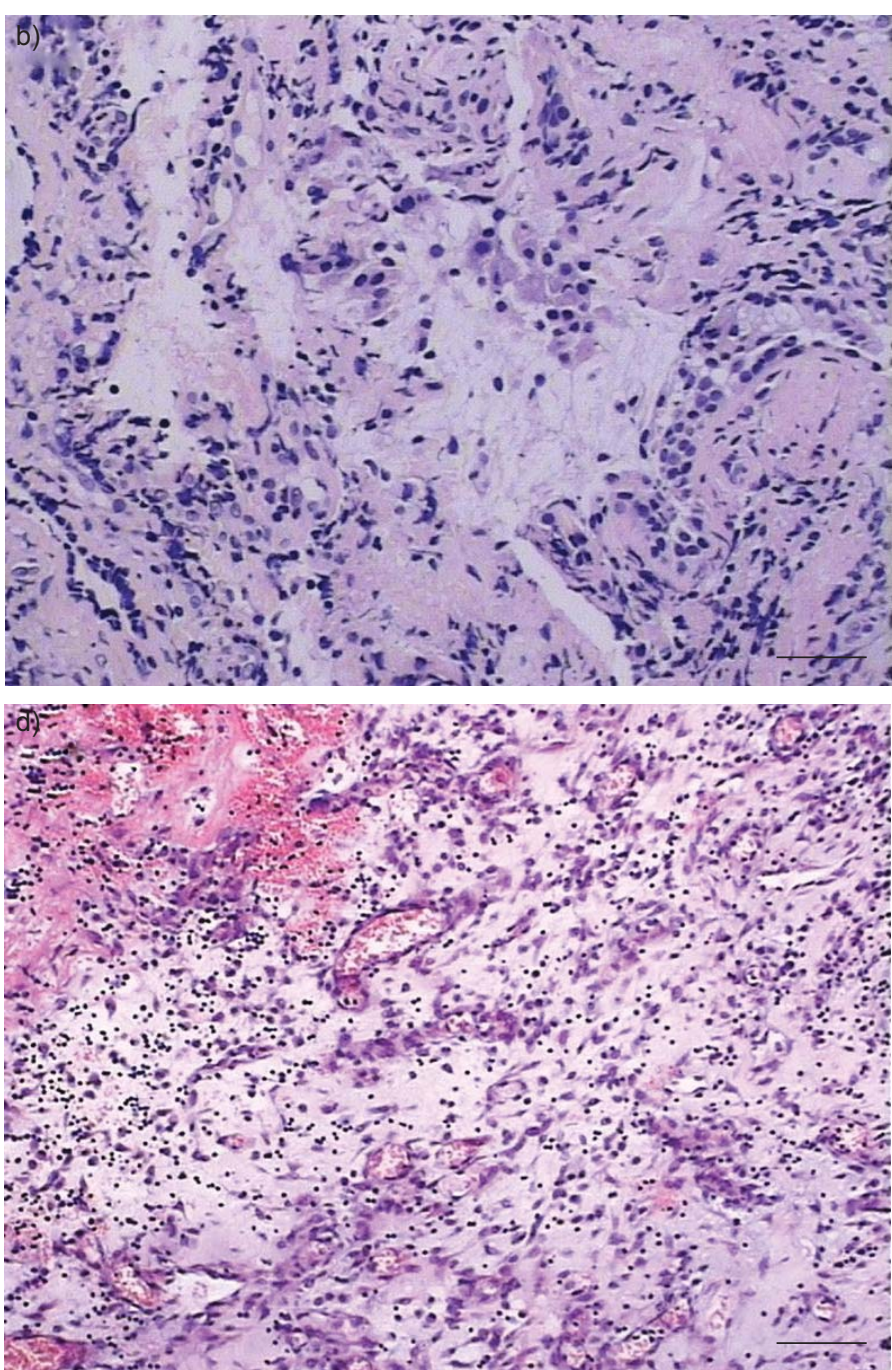

FIGURE 1. a) Aggregation of phagocytes in the alveolar space, swollen and widened alveolar septums, and pulmonary fibrosis were observed (haematoxylineosin (HE) stain). b) Effusion of inflammatory cells was observed in tunica mucosa bronchiorum (HE stain). c) Pathological examination of the pleura showed foreignbody granulomas with fibrinous and inflammatory cell effusions (HE stain). Arrows indicate foreign body giant polykaryocytes. d) Pathological examination of the pleural membrane 18 months later showed fibrous thickening and swelling, fibroblast proliferation, cellulosic exudate and lymphocyte aggregation (HE stain). e) Pulmonary pathological examination 18 months later showed the alveolar septum was widened with blood vessel dilatation and congestion. Pulmonary alveoli were partly emphysematous with scattered multinucleated giant cells (HE stain). a, d) Scale bars $=50 \mu \mathrm{m}$. b, c, e) Scale bars $=25 \mu \mathrm{m}$.

were observed. The pleural membrane was noted to have severe adhesions and thickening, especially in the visceral and diaphragmatic pleura, which measured $1 \mathrm{~cm}$ in thickness and was hard, similar to a cuirass (armour plate). Fenestration of the pericardium was performed, and $170 \mathrm{~mL}$ of light-coloured fluid was drained from the pericardial cavity. 

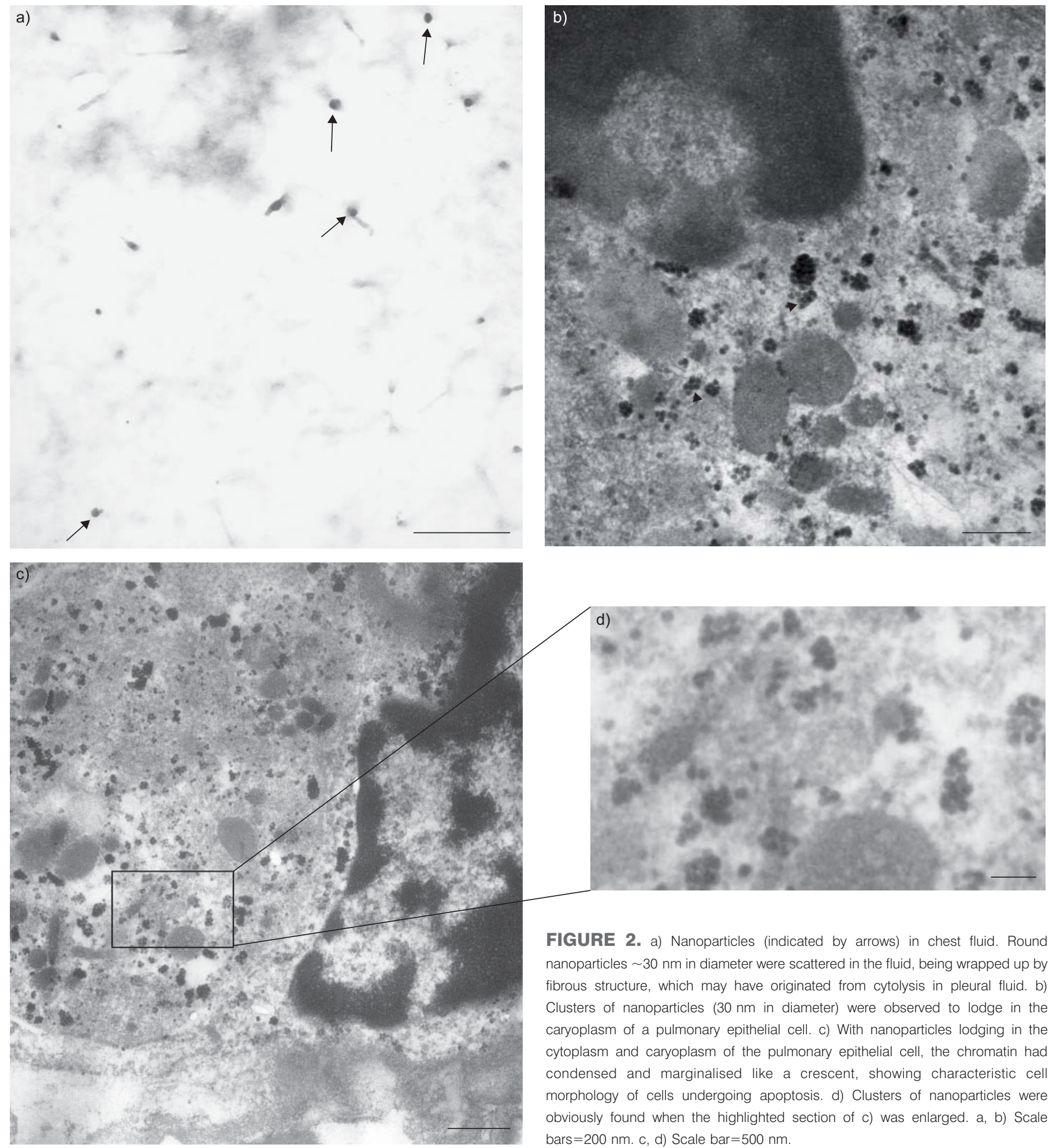

FIGURE 2. a) Nanoparticles (indicated by arrows) in chest fluid. Round nanoparticles $\sim 30 \mathrm{~nm}$ in diameter were scattered in the fluid, being wrapped up by fibrous structure, which may have originated from cytolysis in pleural fluid. b) Clusters of nanoparticles ( $30 \mathrm{~nm}$ in diameter) were observed to lodge in the caryoplasm of a pulmonary epithelial cell. c) With nanoparticles lodging in the cytoplasm and caryoplasm of the pulmonary epithelial cell, the chromatin had condensed and marginalised like a crescent, showing characteristic cell morphology of cells undergoing apoptosis. d) Clusters of nanoparticles were obviously found when the highlighted section of c) was enlarged. a, b) Scale bars $=200 \mathrm{~nm}$. c, d) Scale bar $=500 \mathrm{~nm}$.

Pathological examination (fig. 1d and e) showed fibrous thickening and swelling of the pleural membrane, fibroblast proliferation, cellulosic exudate, lymphocyte aggregation and nodulus lymphaticus formation. The alveolar septum was widened with blood vessel dilatation and congestion. There were type II alveolar epithelial cell proliferations, and pulmonary alveoli were partly emphysematous with scattered multinucleated giant cells.

\section{Examinations of lung pathology and chest fluid by TEM}

Using TEM, round nanoparticles $\sim 30 \mathrm{~nm}$ in diameter were found to scatter in the fluid after a drop was stained by uranyl acetate, air-dried and observed (fig. 2a). The nanopaticles were wrapped up in a fibrous structure, which may have originated from the cytolysis in chest fluid. After the upper liquid of the chest fluid was centrifuged at $123 \times g$, treated as previously 
stated and observed, the nanoparticles looked like sperm or comets because the fibrous structure was not completely separated. In the mesothelial cells of the pleural fluid, similar nanoparticles were found to scatter in the cytoplasm, and lodge in nuclei and other cytoplasmic organoids.

In the lung tissue, nanoparticles were observed to lodge in the cytoplasm and caryoplasm of the pulmonary epithelial cells (fig. 2b), with the chromatin condensation and marginalisation, like a crescent, showing characteristic cell morphology of cells undergoing apoptosis (fig. 2c and d). Additionally, around intravascular red blood cells in the pulmonary interstitial tissue, there was a massive aggregation of nanoparticles. No poikilocytosis was noted. In the BALF, similar nanoparticles were found.

\section{Outcome of the patients}

Two patients suffered from iatrogenic pneumothorax, one secondary to thoracoscopy examination and its lung biopsy, the other due to VATS and the lung biopsy. Both patients recovered quickly after treatment. The rashes and itching on the face and arms were treated symptomatically. Follow-up at 20 months revealed persistent shortness of breath, continued pleural effusions and slowly progressive pulmonary fibrosis.

The pleural effusions of two patients (case 5 and case 1) developed so quickly that continuous closed drainage of the thoracic cavity was necessary $(300-800 \mathrm{~mL}$ pleural fluid was usually drained everyday). VATS was performed as an emergency on the 19-yr-old female (case 5) 18 months after symptom onset. The procedure included fenestration of the pericardium, decortication of pleural fibrous lamina, wedge resection, lung biopsies, and drainage of pleural effusion. Thereafter, she suffered from severe tension pneumothorax $24 \mathrm{~h}$ post-VATS, and acute pericardial tamponade on the 10th day post-surgery, which were all successfully treated. The patient died of respiratory failure 16 days post-surgery. A 29yr-old female (case 1), who was similar to the 19-yr-old female (case 5) both in clinical findings and the development of the illness, died of respiratory failure in local hospital 21 months after symptom onset.

\section{DISCUSSION}

From the report, the patients have several common characteristics. First, they were all young females who were exposed to nanoparticles consisting of polyacrylic ester without any protective measures for 5-13 months. Also, they presented in the same time frame with the same symptoms: shortness of breath, and the same clinical findings of pleural and pericardial effusions consisting of an amber exudate, which may be produced secondary to physical or chemical irritation. These effusions recurred repeatedly after thoracentesis, and other treatments were rendered ineffective. Additionally, the pathological examinations displayed nonspecific pulmonary inflammation, inflammatory infiltration, pulmonary fibrosis, and foreign-body granulomas of the pleura. These findings were consistent with damage produced by nanoparticles in animal experiments $[7,9]$. Importantly, nanoparticles $\sim 30 \mathrm{~nm}$ in diameter were found in the used paste and dust particles of the workplace as well as in the BALF, chest fluid and lung biopsies of the patients. Using TEM, nanoparticles were found to lodge in the cytoplasm and caryoplasm of the pulmonary epithelial and mesothelial cells of the chest fluid. Also, the chromatin of the pulmonary epithelial cell condensed and marginalised like a crescent, showing characteristic cell morphology of cells undergoing apoptosis. In all of these cases, clinical observations and examinations excluded infections, malignant tumours, immune-related disorders and other diseases.

We could infer that the patients may have suffered from damage related to nanoparticles ( $30 \mathrm{~nm}$ in diameter) by comparing these cases with the toxicity of nano materials observed in the animal experiments. These animal studies showed nonspecific pulmonary inflammation, granuloma formation of lungs, rapidly progressive fibrosis and lung function deficiency following nano materials aspiration [7-9].

As observed in animal and in vitro experiments, nano materials are found to cause disorders of blood element tests and hepatic function tests of serum ALT and AST [10,11]. In the group of patients, five patients had monocytosis with an elevated absolute monocyte count and neutropenia with a decreased absolute neutrophil count; additionally, five patients had elevated ESR and one patient had thrombocytopenia. Moreover, several patients were found with elevated levels of ALT and AST. The observations above suggest the haematological toxicity of the polyacrylate nanoparticles. However, the hypoalbuminaemia found in some patients is probably due to prolonged drainage of pleural fluid rather than a specific effect of nanoparticle exposure, as the levels of serum album in patients were all in the NR in the beginning and, furthermore, no hypoalbuminaemia related to nanoparticles has been reported in animal experiments.

Polyacrylate, widely used as an adhesive in the building, print and decoration fields, has often been regarded as low toxicity. To make the material stronger and more abrasion-resistant, nanoparticles, after being modified to turn their surface from hydrophilic to organophilic, can be directly added in organic resin to make organic-inorganic hybrids. These inorganics include silicon nanoparticles [12], thin zinc oxide [13], titanium dioxide $\left(\mathrm{TiO}_{2}\right)$ [14], nanoscale silver cluster [15], and other engineered nano materials. A series of silicon-containing polyacrylate nanoparticles have also been successfully synthesised and widely used [12, 16]. However, it is the nano materials containing nano-size particles that appear to produce the toxicities seen in the exposed workers.

Therefore, we have more evidence to show that the nanoparticles contained in the polyacrylate emulsion had possibly caused the disease. There is an indication from this report that shows the possible dangerous nature of nanoparticles. Nanoparticles can penetrate the membrane of pulmonary epithelial cells and lodge in the cytoplasm and caryoplasm, as well as aggregate around the membrane of red blood cells and exert toxicity. Patients may develop clinically serious conditions associated with damaged respiratory function, including a progressive pulmonary fibrosis that is resistant to several methods of treatment.

Animal studies show that some nanoparticles can travel throughout the body, deposit in target organs, penetrate cell membranes, lodge in mitochondria, and trigger injurious responses. Inflammatory and granulomatous responses are 
seen in the lungs following exposure to carbon nanotubers [7, $17,18]$. A rapid progressive fibrosis in mice exhibited diffuse interstitial fibrosis and alveolar wall thickening likely associated with dispersed nanoparticles [7]. The major routes for nanoparticles to enter the body may be through inhalation, ingestion, dermal and injection [4]. In this group of patients, we could infer that exposure to nanoparticles occurred at least by inhalation and dermal routes.

\section{Inhalation}

The respiratory system is one of the most important portals of entry and organ target for nanoparticles, which can avoid normal phagocytic defences in the respiratory system and travel throughout the pulmonary alveoli. Once inhaled and deposited, nanoparticles appear to translocate to extra pulmonary sites and reach other target organs by different transfer routes and induce toxicological effects $[4,19]$. The generation of smoke at the workplace when the PS board is heated and dried may indicate the formation of a condensation aerosol (containing nanoparticles) from volatilised material. Particularly, as the freshly generated fumes are highly reactive and damaging [20], the nanoparticles entered the body while the spraying, heating and drying of the boards were being performed may exert great toxicities on the exposure workers. In addition, this could help to explain the nanoparticles deposition in the respiratory tract and in BALF, formation of lung fibrosis, occurrence of multi-serous membrane fluid due to the irritation of nanoparticles to cell membrane, existence of foreign body granulomas in pleura, inflammatory infiltration and hilar lymphadenopathy.

\section{Dermal}

Studies of nanoparticle absorption through the skin are inconclusive and disputable, some demonstrate little penetration into the epidermis while others show deep absorption [2123]. Alvarez-Roman et al. [24] show that although polystyrene nanoparticles (20-200 nm) accumulate in the follicle orifices, the particles do not penetrate into the skin or the follicle. However, some studies suggest that skin is surprisingly permeable to nano materials with diverse physicochemical properties, and may serve as a portal of entry for localised or possibly systemic exposure of humans to engineered nano materials $[25,26]$. Nanoparticles can penetrate intact skin at an occupationally relevant dose within the span of an averagelength workday. A recent study on $\mathrm{TiO}_{2}$ nanoparticles at the workplace found that $\mathrm{TiO}_{2}$ nanoparticles production workers have significant risk on cytotoxicity response at relatively high airborne concentrations at size range $10-30 \mathrm{~nm}$ [27]. Furthermore, protein tyrosine nitration is a potential hazard of nano $\mathrm{TiO}_{2}$ on the skin, the toxicity of nano $\mathrm{TiO}_{2}$ to skin disease should be paid more attention to in the production and utilisation process [28]. In this group of patients, skin may be an available portal of entry into the body by occupational dermal exposure; this could help to explain the rash and itching on the exposed skin of our patients, probably the dermal toxicity of the polyacrylate nanoparticles.

The kind of the nanoparticles and the mechanisms of the injury in patients have not been firmly established. However, animal studies and in vitro experiments may provide a clue that the nanoparticles themselves [6-8], not as carriers, cause the injury directly; although, the nanoparticles may also act as carriers facilitating translocation and persistence in the pleural and pericardial spaces. Furthermore, after being analysed by GC/ MS, the compounds in the materials used in the workplace, such as butanoic acid, acetic acid, toluene and ethylene dioxide, are low in toxicity and are unlikely to cause disease of the severity seen in these patients. Thus, the patients' illness appears to be a "nano material-related disease".

There are several limitations about our study. First, this description of patients is limited by the absence of environmental monitoring data of the workplace. Because no one suspected that the illness of the patients would be related to the occupational nanoparticles exposure a very long time after the occurrence of the accident, the environmental monitoring of the workplace was not performed in time and the accurate concentrations of the polyacrylate nanoparticles that the workers were probably exposed to was still not known. However, the detailed description of their working, the duration of daily exposure, the dosage of the material used everyday, the space of their workplace and the serious results of long-term exposure give us some important information that the concentrations of the polyacrylate nanoparticles that the workers were exposed to may be very high. Secondly, the composition of the nanoparticles is still unknown, although a lot of efforts have been made including contacting the manufacturer concerning the content of nano materials in the product. Thirdly, there are still many questions to be answered. What are the nanoparticles (chemistry and composition) found in patients? Would only specific nanoparticles, or nanoparticles in general, cause the illness in patients? If it is the nanoparticles that caused the patients' disease, what does it mean for other workers who use nanoparticles in their workplace? Was this an isolated incident or could this be occurring on a larger scale? What are the effects of long-term exposure to nanoparticles in animal studies? However, all data given in the study have suggested that the polyacrylate nanoparticles that the patients were exposed to are linked to the patients' illnesses. As this may be the first study on the clinical toxicity in humans due to long-term exposure to nanoparticles, and so many questions need to be answered, more studies on the possible mechanisms, diagnosis, treatment and prevention of the "nano material-related disease" are needed.

In conclusion, these cases arouse concern that long-term exposure to some nanoparticles without protective measures may be related to serious damage to human lungs. It is impossible to remove nanoparticles that have penetrated the cell and lodged in the cytoplasm and caryoplasm of pulmonary epithelial cells, or that have aggregated around the red blood cell membrane. Effective protective methods appear to be extremely important in terms of protecting exposed workers from illness caused by nanoparticles.

\section{SUPPORT STATEMENT}

The present study was funded, in part, by the China National Key Project (2008ZX10003-009; Beijing, China).

\section{STATEMENT OF INTEREST}

None declared. 


\section{ACKNOWLEDGEMENTS}

The current authors would like to thank the doctors and nurses from the Depts of Pulmonary Medicine, Thoracic Surgery, Pathology and Occupational Medicine (Beijing Chaoyang Hospital, Capital University of Medical Sciences, Beijing, China) for their hard work in the treatment of the patients; and M. Spartz (Neck and Back Clinic, Minneapolis, MN, USA) for reading the manuscript for clarity.

\section{REFERENCES}

1 Roco MC. International perspective on government nanotechnology funding in 2005. J Nanopart Res 2005; 7: 707-712.

2 Kipen HM, Laskin DL. Smaller is not always better: nanotechnology yields nanotoxicology. Am J Physiol Lung Cell Mol Physiol 2005; 289: L696-L697.

3 Nel A, Xia T, Madler L, et al. Toxic potential of materials at the nano level. Science 2006; 311: 622-627.

4 Oberdörster G, Oberdörster E, Oberdörster J. Nanotoxicology: an emerging discipline evolving from studies of ultrafine particles. Environ Health Perspect 2005; 113: 823-839.

5 Seaton A, Donaldson K. Nanoscience, nanotoxicology, and the need to think small. Lancet 2005; 365: 923-924.

6 Papageorgiou I, Brown C, Schins R, et al. The effect of nano- and micron-sized particles of cobalt-chromium alloy on human fibroblasts in vitro. Biomaterials 2007; 28: 2946-2958.

7 Shvedova AA, Kisin ER, Mercer R, et al. Unusual inflammatory and fibrogenic pulmonary responses to single-walled carbon nanotubes in mice. Am J Physiol Lung Cell Mol Physiol 2005; 289: L698-L708.

8 Hamilton RF, Buford MC, Wood MB, et al. Engineered carbon nanoparticles alter macrophage immune function and initiate airway hyper-responsiveness in the BALB/C mouse model. Nanotoxicology 2007; 1: 104-117.

9 Lam CW, James JT, McCluskey R, et al. Pulmonary toxicity of single-wall carbon nanotubes in mice 7 and 90 days after intratracheal instillation. Toxicol Sci 2004; 77: 126-134.

10 Wang B, Feng WY, Wang TC, et al. Acute toxicity of nano- and micro-scale zinc powder in healthy adult mice. Toxicol Lett 2006; 161: 115-123.

11 Longest PW, Kleinstreuer C. Comparison of blood particle deposition models for non-parallel flow domains. J Biomech 2003; 36: $421-430$.

12 Mizutani T, Arai K, Miyamoto M, et al. Application of silicacontaining nano-composite emulsion to wall paint: a new environmentally safe paint of high performance. Progr Org Coating 2006; 55: 276-283.

13 Ali HA, Iliadis AA. Thin $\mathrm{ZnO}$ nanocomposite poly(styrene-acrylic acid) films on $\mathrm{Si}$ and $\mathrm{SiO}_{2}$ surfaces. Thin Solid Films 2005; 471: 154-158.
14 Chen Y, Lin A, Gan F. Improvement of polyacrylate coating by filling modified nano- $\mathrm{TiO}_{2}$. Appl Surf Sci 2006; 252: 8635-8640.

15 Mitra A, Bhaumik A. Nanoscale silver cluster embedded in artificial heterogeneous matrix consisting of protein and sodium polyacrylate. Mater Lett 2007; 61: 659-662.

$16 \mathrm{Ma} \mathrm{JZ,} \mathrm{Hu} \mathrm{J,} \mathrm{Zhang} \mathrm{ZJ.} \mathrm{Polyacrylate/silica} \mathrm{nanocomposite}$ materials prepared by sol-gel process. Eur Polym J 2007; 43: 4169-4177.

17 Medina C, Santos-Martinez MJ, Radomski A, et al. Nanoparticles: pharmacological and toxicological significance. $\mathrm{Br} J$ Pharmacol 2007; 150: 552-558

18 Oberdörster G, Maynard A, Donaldson K, et al. Principles for characterizing the potential human health effects from exposure to nanomaterials: elements of a screening strategy. Part Fibre Toxicol 2005; $2: 8$.

19 Zhu MT, Feng WY, Wang Y, et al. Particokinetics and extrapulmonary translocation of intratracheally instilled ferric oxide nanoparticles in rats and the potential health risk assessment. Toxicol Sci 2009; 107: 342-351.

20 Antonini JM, Clarke RW, Murthy GGK, et al. Freshly generated stainless steel welding fume induces greater lung inflammation in rats as compared to aged fume. Toxicol Lett 1998; 98: 77-86.

21 Hagens WI, Oomen AG, de Jong WH, et al. What do we (need to) know about the kinetic properties of nanoparticles in the body? Regul Toxicol Pharmacol 2007; 49: 217-219.

22 Tinkle SS, Antonini JM, Rich BA, et al. Skin as a route of exposure and sensitization in chronic beryllium disease. Environ Health Perspect 2003; 111: 1202-1208.

23 Rouse JG, Yang J, Ryman-Rasmussen JP, et al. Effects of mechanical flexion on the penetration of fullerene amino acidderivatized peptide nanoparticles through skin. Nano Lett 2007; 7: 155-160.

24 Alvarez-Roman R, Naik A, Kalia YN, et al. Skin penetration and distribution of polymeric nanoparticles. J Control Release 2004; 99 : 53-62.

25 Baroli B, Ennas MG, Loffredo F, et al. Penetration of metallic nanoparticles in human full-thickness skin. J Invest Dermatol 2007; 127: 1701-1712.

26 Ryman-Rasmussen JP, Riviere JE, Monteiro-Riviere NA. Penetration of intact skin by quantum dots with diverse physicochemical properties. Toxicol Sci 2006; 91: 159-165.

27 Liao $\mathrm{CM}$, Chiang $\mathrm{YH}$, Chio $\mathrm{CP}$. Assessing the airborne titanium dioxide nanoparticle-related exposure hazard at workplace. J Hazard Mater 2009; 162: 57-65.

$28 \mathrm{Lu} \mathrm{N}, \mathrm{Zhu} \mathrm{Z}$, Zhao X, et al. Nano titanium dioxide photocatalytic protein tyrosine nitration: a potential hazard of $\mathrm{TiO}_{2}$ on skin. Biochem Biophys Res Commun 2008; 370: 675-680. 